\title{
Clinical impact and predictors of carotid artery in-stent restenosis
}

\author{
Katrin Wasser • Sonja Schnaudigel • \\ Janin Wohlfahrt • Marios-Nikos Psychogios • \\ Peter Schramm • Michael Knauth • Klaus Gröschel
}

Received: 4 January 2012 / Revised: 16 January 2012/ Accepted: 20 January 2012/Published online: 9 February 2012

(C) The Author(s) 2012. This article is published with open access at Springerlink.com

\begin{abstract}
To assess the incidence and clinical significance as well as predictors of in-stent restenosis (ISR) after carotid artery stenting (CAS) diagnosed with serial duplex sonography investigations. We analyzed 215 CAS procedures that had clinical and serial carotid duplex ultrasound investigations. The incidence of in-stent restenosis (ISR) and periprocedural as well as long-term clinical complications were recorded. The influence of an ISR on clinical complication was analyzed using Kaplan-Meier curves and clinical risk factors for the development of an ISR with multivariate logistic regression. During a median follow-up time of 33.4 months (interquartile range 15.3-53.7) an ISR of $\geq 70 \%$ was detected in $12(6.1 \%)$ of 215 arteries (mean age of $68.1 \pm 9.8$ years, $71.6 \%$ male). The combined stroke and death rate during long-term follow-up was significantly higher in the group with an ISR [odds ratio (OR): $3.59,95 \%$ confidence interval (CI): $1.50-8.59, p=0.004]$. After applying multivariate logistic regression analysis contralateral carotid occlusion (OR 10.11, 95\% CI 2.06-49.63, $p=0.004$ ), carotid endarterectomy (CEA) restenosis (OR 8.87, 95\% CI 1.68-46.84, $p=0.010)$ and postprocedural carotid duplex ultrasound with a PSV $\geq 120 \mathrm{~cm} / \mathrm{s}$ (OR 6.33, 95\% CI 1.27-31.44, $p=0.024$ )
\end{abstract}

K. Wasser · S. Schnaudigel · J. Wohlfahrt · K. Gröschel Department of Neurology, University of Göttingen, Robert-Koch-Str. 40, 37075 Göttingen, Germany

M.-N. Psychogios · P. Schramm · M. Knauth Department of Neuroradiology, University of Göttingen, Robert-Koch-Str. 40, 37075 Göttingen, Germany

K. Gröschel ( $\square)$

Department of Neurology, University of Mainz, Langenbeckstr. 1, 55131 Mainz, Germany

e-mail: klaus.groeschel@unimedizin-mainz.de were independent predictors of ISR. ISR after CAS during long-term follow-up is associated with a higher proportion of clinical complications. A close follow-up is suggested especially in those patients with the aforementioned independent predictors of an ISR. Against the background of a lacking established treatment of ISR, these findings should be taken into account when offering CAS as a treatment alternative to CEA.

Keywords Carotid artery stenosis - Stent · Angioplasty · Restenosis $\cdot$ Stroke $\cdot$ Duplex sonography

\section{Introduction}

Atherosclerotic stenosis of the carotid artery is known as a major risk factor for ischaemic stroke. Carotid endarterectomy (CEA) in combination with best medical treatment of cerebrovascular risk factors is considered to be the gold standard for primary and secondary stroke prevention in patients with significant carotid artery stenosis. More recently, carotid angioplasty and stenting (CAS) have emerged as a potentially less invasive treatment alternative. However, the results of randomized controlled studies and subsequent meta-analyses comparing CEA with CAS failed to prove a general superiority of CAS [1-3]. Nevertheless, there is growing evidence that a subgroup of patients aged $<70$ years may benefit from CAS intervention [3, 4]. A current major drawback is that prospective data with respect to the clinical long-term outcome are sparse and controversially discussed [1]. Especially the occurrence of an in-stent restenosis (ISR) could endanger the long-term efficacy and safety of CAS. Considering the fact that to date there is no established treatment strategy for an ISR, this issue will be of high clinical importance particularly if 
patients $<70$ years are preferably being treated with CAS in the future. By now, the exact rate and clinical impact of ISR during long-term follow-up is still unclear, which may in part be attributable to different definitions of the duplex criteria of an ISR during follow-up investigations [5, 6].

Therefore, the current study had three major aims: first, to investigate the incidence of ISR as assessed with serial duplex ultrasonography; second, to evaluate the impact of ISR on clinical complications (stroke or death) during long-term follow-up; third, to analyze clinical predictors for ISR in order to identify patients at greatest risk who are expected to benefit from a rigorous follow-up.

\section{Methods}

\section{Patients}

Within a prospectively created single-center CAS database we conducted a retrospective analysis of a total of 198 patients ( 215 arteries) that had been treated between May 2003 and June 2010. Patients had undergone a CAS intervention because of a high-grade carotid stenosis defined as $\geq 70 \%$ in symptomatic patients and $\geq 90 \%$ in asymptomatic patients according to the European Carotid Surgery Trial (ECST) criteria. A carotid stenosis was considered symptomatic if the patient had experienced a transient or permanent ipsilateral ocular or cerebral ischemic event within the past 6 months. All patients received information about the different treatment modalities (CEA, CAS and best medical treatment) and their respective advantages and disadvantages, in particularly the potential complications and risks. With respect to the CAS procedure, all patients were informed about the investigational nature of CAS and gave their written informed consent. The current study is in accordance with International Conference on Harmonisation/Good Clinical Practice guidelines and was approved by the local ethics committee.

\section{Data collection}

An experienced stroke neurologist documented clinical data potentially responsible for influencing the occurrence of an ISR at every follow-up visit. The etiology of all stenoses was atherosclerotic and was further subdivided into a naïve carotid stenosis or a post-endarterectomy stenosis (CEA restenosis). The following cerebrovascular risk factors were recorded using history or direct measurements: hypertension (blood pressure $\geq 140 / 90 \mathrm{mmHg}$ measured on repeated occasions or presence of antihypertensive drugs), hyperlipidemia (fasting serum cholesterol levels $\geq 200 \mathrm{mg} / \mathrm{dl}$ or statin therapy), diabetes mellitus (HbA1c $\geq 6.5 \%$, fasting blood glucose $\geq 120 \mathrm{mg} / \mathrm{dl}$ or presence of antidiabetic drugs), smoking (current or within the previous year), coronary artery disease (history of angina, myocardial infarction, percutaneous transluminal angioplasty or surgery), peripheral occlusive arterial disease (history of typical clinical presentation, percutaneous transluminal angioplasty or surgery) and the presence of contralateral carotid disease (as assessed with ultrasound and subdivided into a stenosis $\geq 70 \%$ or occlusion).

The periprocedural 30-day complications were recorded and categorized as stroke (any neurological deficit persistent $>24 \mathrm{~h}$ ) or death (death of any cause). Long-term follow-up complications recorded in the current study were ipsilateral (symptoms corresponding to the treated artery) stroke or death from any cause. Furthermore, the date and character of carotid re-interventions (balloon angioplasty alone, CAS or CEA) and their specific complications (any stroke or death within 30 days of the procedure) were registered.

\section{CAS procedure}

CAS was carried out by experienced interventional neuroradiologists and done under anesthesiological stand-by. All interventions were performed via a transfemoral approach. Stent type and the use of filter-based neuroprotection devices were chosen at the discretion of the interventionalist. Only patients scheduled for elective CAS were recorded; patients in unstable clinical conditions or with stroke in evolution were excluded. All patients received orally administered acetylsalicylic acid $(100 \mathrm{mg} /$ day) and clopidogrel (75 mg/day) at least 3 days before the procedure. Clopidogrel was continued for a minimum of 6 weeks after CAS, and aspirin was administered indefinitely. After being routinely monitored in our intensive care or stroke unit overnight for at least 1 day, all patients were discharged afterwards to a normal ward or home. A clinical examination and duplex sonography were performed before discharge to obtain the clinical status of the patient and confirm stent patency.

\section{Doppler and duplex sonography}

The diagnosis of a carotid artery stenosis and an ISR in particular was made by carotid duplex ultrasound imaging using a combination of direct and indirect criteria, and the presence and extent of intrastenotic and poststenotic turbulent flow. In detail, as direct criteria for the local degree of stenosis, the peak systolic flow velocities (PSV) within the stenosis and poststenotic internal carotid artery, the end diastolic flow velocity in the stenosis, the internal carotid artery/common carotid artery PSV ratio, and the prestenotic and poststenotic frequency patterns were determined. The residual vessel lumen in the B image and the color- 
coded residual vessel area were documented whenever possible. The flow characteristics of the supratrochlear artery and the anterior cerebral artery as well as the pulsatility of the ipsilateral common carotid artery were taken into account as indirect criteria for a higher grade stenosis. As one of the main criteria the degree of carotid stenosis at baseline was graded according to angle-corrected maximum intrastenotic peak systolic velocities according to ECST criteria as follows: baseline stenosis $\geq 70 \%=\mathrm{PSV}$ $\geq 200 \mathrm{~cm} / \mathrm{s}$, baseline stenosis $\geq 80 \%=\mathrm{PSV} \geq 300 \mathrm{~cm} / \mathrm{s}$, baseline stenosis $\geq 90 \%=\mathrm{PSV} \geq 400 \mathrm{~cm} / \mathrm{s}$.

As there is a lack of generally valid ultrasound criteria for the definition of an ISR and the current literature supposes different criteria [5, 6], we used locally adopted criteria with a PSV $\geq 300 \mathrm{~cm} / \mathrm{s}$ as a key feature representing an ISR of $\geq 70 \%$.

All examinations were performed according to a standardized protocol in the same vascular laboratory with the same ultrasound equipment (Acuson Sequoia ${ }^{\mathrm{TM}}$ 512, Siemens, San José, CA) under the supervision of an experienced, board-certified vascular neurologist (K.G.).

\section{Follow-up protocol}

All patients were summoned for serial duplex sonography follow-up at the hospital's outpatient clinic at 3, 6 and 12 months after the CAS procedure and every 6 months thereafter. During these routine postinterventional visits, a neurologist experienced in neurovascular diseases examined each patient and recorded the aforementioned clinical complications.

\section{Statistical analysis}

Nominal variables were expressed as count and percentages, continuous values as mean \pm standard deviation (SD) and not normally distributed values as median values with the corresponding interquartile range (IQR), respectively. For univariate comparisons of categorical data, twotailed chi-square statistics with Yates' correction and univariate Fisher's exact test were used. The Fisher's exact test was applied when the predicted contingency table cell values were less than 5 . Non-normally distributed variables were compared using a Mann-Whitney $U$ test.

Overall survival Kaplan-Meier curves were obtained using periprocedural ( $\leq 30$ days) stroke and death as well as ipsilateral stroke and death during long-term follow-up (>30 days) as a combined endpoint. Interaction for the occurrence of an ISR was tested using the Mantel-Cox test. In order to estimate a potential effect of a variable on the occurrence of an ISR during follow-up, we used a multiple binominal regression analysis. All variables with a $p<0.1$ on the univariate level were included into a multiple binominal regression analysis ( $p$ to enter $=0.05, p$ to leave $=0.1)$. A two-sided $p$ value of less than 0.05 was considered to indicate a statistically significant difference. All statistical analyses were performed with SPSS (version 17, SPSS Inc., Chicago, IL).

\section{Results}

Patient characteristics

Two hundred nineteen patients (237 arteries) undergoing elective carotid artery stenting between May 2003 and June 2010 were analyzed. The data of 21 patients (22 arteries) had to be excluded because of a missing Duplex follow-up, yielding a total follow-up rate of $90.7 \%$. Complete clinical follow-up data with a median duration of 33.4 months (IQR 15.0-53.7) were available for all the remaining 215 arteries (mean age of $68.1 \pm 9.8$ years, $71.6 \%$ male).

The detailed patient characteristics are given in Table 1 . An ISR $\geq 70 \%$ with a PSV $\geq 300 \mathrm{~cm} / \mathrm{s}$ was detected in $12 / 215(5.6 \%)$ arteries in 198 patients after a median of 8.6 months (IQR 3.4-17.3). In 9/12 patients (75\%) a retrograde flow of the ipsilateral supratrochlear artery and/or the anterior cerebral artery could be detected as indirect duplex criteria of a high grade ISR. Contrast-enhanced reference imaging was performed in $10 / 12$ cases $(83.3 \%)$. A higher grade ISR could be confirmed in $9 / 10$ patients (90\%, 7 by digital subtraction angiography and 2 by computer tomography angiography).

Considering the development of an ISR, the cardiovascular risk factors hyperlipidemia and peripheral artery occlusion disease as well as an intervention because of a CEA restenosis in comparison to a naïve CAS procedure were statistically significantly more frequent in this group on univariate level ( 91.7 vs. $65.0 \%, p=0.07 ; 41.7$ vs. $18.2 \%, p=0.061 ; 33.3$ vs. $7.4 \%, p=0.014$, respectively). Moreover, patients with an ISR during follow-up more often presented with a contralateral occlusion or stenosis of the ICA $\geq 70 \%$ at the time of CAS and a post-interventional flow acceleration with a PSV $\geq 120 \mathrm{~cm} / \mathrm{s}(41.7$ vs. $11.3 \%, p=0.01 ; 58.3$ vs. $24.6 \%, p=0.02 ; 33.3$ vs. $5.9 \%$, $p<0.001)$.

\section{Clinical complications}

During the 30-day periprocedural follow-up time, the overall stroke and death rate was $7.4 \%$. Twelve patients $(5.6 \%)$ suffered from stroke and five patients $(2.3 \%)$ died after a median of 3 days (IQR 3-14). One patient had discontinued his antithrombotic medication and subsequently died of a major stroke after developing an acute instent thrombosis. One patient died of an intracerebral 

characteristics of the study population
Table 1 Baseline
* Factors included in multiple regression analysis $(p<0.1$ univariate analysis)

${ }^{\dagger}$ Factors remained significant after multiple regression analysis

\begin{tabular}{|c|c|c|c|}
\hline \multirow[t]{2}{*}{ Variable } & \multicolumn{3}{|l|}{ Data } \\
\hline & No ISR & ISR $\geq 70 \%$ & $p$ value \\
\hline$N$ & 203 & 12 & \\
\hline Age (years) & $68.1 \pm 9.8$ & $67.8 \pm 6.6$ & 0.928 \\
\hline Male sex & $147(72.4 \%)$ & $7(58.3 \%)$ & 0.328 \\
\hline Weight $(\mathrm{kg})$ & $79.4 \pm 13.4$ & $82.5 \pm 6.6$ & 0.661 \\
\hline Height (m) & $170.6 \pm 7.6$ & $169.5 \pm 3.9$ & 0.782 \\
\hline Left side & $115(56.7 \%)$ & $4(33.3 \%)$ & 0.141 \\
\hline Symptomatic carotid stenosis & $154(75.9 \%)$ & $7(58.3 \%)$ & 0.328 \\
\hline Stroke & $94(46.3 \%)$ & $3(25.0 \%)$ & 0.232 \\
\hline Hemispherical TIA & $45(22.2 \%)$ & $3(25.0 \%)$ & 0.733 \\
\hline Amaurosis fugax & $8(3.9 \%)$ & $1(8.3 \%)$ & 0.410 \\
\hline Hypertension & $184(90.6 \%)$ & $12(100 \%)$ & 0.606 \\
\hline Hyperlipidemia & $132(65.0 \%)$ & $11(91.7 \%)$ & $0.065^{*}$ \\
\hline Diabetes & $61(30.0 \%)$ & $2(16.7 \%)$ & 0.516 \\
\hline Tobacco use & $59(29.1 \%)$ & $5(41.7 \%)$ & 0.347 \\
\hline Coronary artery disease & $60(29.6 \%)$ & $4(33.3 \%)$ & 0.753 \\
\hline Peripheral occlusive arterial disease & $37(18.2 \%)$ & $5(41.7 \%)$ & $0.061^{*}$ \\
\hline CEA restenosis & $15(7.4 \%)$ & $4(33.3 \%)$ & $0.014 *$, \\
\hline Contralateral ICA occlusion & $23(11.3 \%)$ & $5(41.7 \%)$ & $0.011^{*}$, \\
\hline Contralateral ICA stenosis $\geq 70 \%$ & $50(24.6 \%)$ & $7(58.3 \%)$ & $0.017 *$ \\
\hline Stenosis $\geq 90 \%$ before CAS & $86(42.4 \%)$ & $6(50.0 \%)$ & 0.603 \\
\hline $\mathrm{PSV} \geq 120 \mathrm{~cm} / \mathrm{s}$ after CAS & $12(5.9 \%)$ & $4(33.3 \%)$ & $0.001^{*}$, \\
\hline Any Stroke or death $\leq 30$ days & $14(6.9 \%)$ & $2(16.7 \%)$ & 0.221 \\
\hline Ipsilateral stroke or death $>30$ days & $22(10.8 \%)$ & $4(33.3 \%)$ & 0.043 \\
\hline Median follow-up time (month, IQR) & $33.4(15.5-53.9)$ & $20.8(5.9-41.8)$ & 0.218 \\
\hline Re-interventions & $0(0 \%)$ & $8(66.7 \%)$ & $<0.001$ \\
\hline
\end{tabular}

hemorrhage immediately after CAS. Other causes of death were a traumatic subarachnoid hemorrhage after discharge, a hypopharynx carcinoma, and one patient died of unknown cause. The combined periprocedural stroke and death rate was $6.9 \%$ in patients without and $16.7 \%$ in those with ISR $(p=0.22)$.

During the long-term follow-up period ( $>30$ days after CAS), the combined rate of ipsilateral stroke and death was $10.8 \%$ in the subgroup without ISR (7 strokes, 1 stroke followed by subsequent death and 14 deaths) and 33.3\% in the group with an ISR $\geq 70 \%$ ( 2 strokes and 2 deaths; $p=0.043$ ) after a median of 31.9 months follow-up time (IQR 16.1-43.3). The cumulative rate of stroke-free survival for patients with and without ISR $\geq 70 \%$ is shown in Fig. 1. Within the patients with ISR there was a statistically significantly higher risk for clinical complications (ipsilateral stroke and death) during follow-up [odds ratio (OR): $3.59,95 \%$ confidence interval (CI): $1.50-8.59, p=0.004]$. For the whole study group Kaplan-Meier analysis estimates the freedom of a $\geq 70 \%$ ISR of $96,95,94,93$ and $91 \%$ after each year.

Within the patients with a restenosis the number of ipsilateral re-interventions during follow-up was

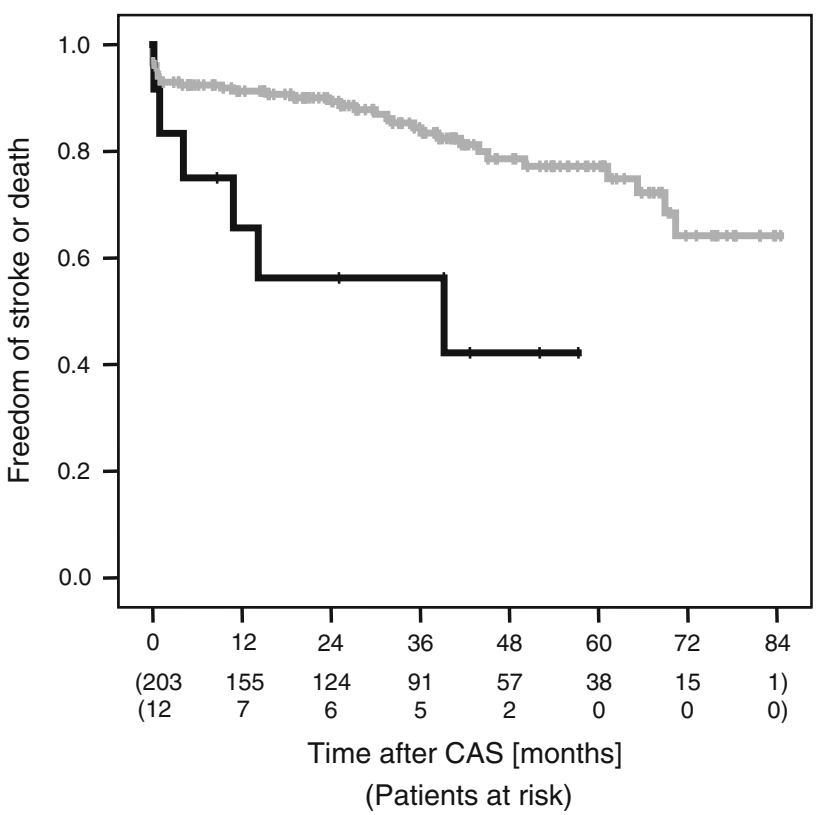

Fig. 1 Kaplan-Meier curve representing the freedom of clinical complications (periprocedural any stroke or death and ipsilateral stroke or any death beyond 30 days) patients with (black) and without (grey) restenosis during follow-up $(p=0.004)$ 
significantly higher (0 vs. $66.7 \%, p<0.001)$ as compared to those without ISR. In the group with ISR $\geq 70 \%$ an ipsilateral re-intervention was performed in eight patients (3 CAS, 4 PTA, 1 bypass; 66.7\%), whereas no interventions of the contralateral side were recorded. Despite the failure of the attempt to recanalize the acute in-stent thrombosis as reported above and the patient's death 3 days later of a major stroke, there were no other periprocedural complications associated with a re-intervention recorded.

\section{Carotid stent procedure}

A detailed description of the procedure has been described recently [7]. In the vast majority a Carotid Wallstent Monorail $^{\circledR}$ ( $n=179$, Boston Scientific Corp., Natick, MA, USA) was deployed followed by the use of a Zilver ${ }^{\circledR}$ ( $n=13$; Cook Medical Inc., Bloomington, IN, USA) or Precise ${ }^{\circledR}$ stent ( $n=11$, Cordis Endovascular, Warren, NJ, USA). Distal filter-type embolic protection systems were used in 61 of $215(28.4 \%)$ patients. No differences were observed among different stent types, open versus closed cell design or the use of a protection system between the two groups. There was a higher proportion of post-procedural residual stenosis as assessed with duplex sonography (PSV $\geq 120 \mathrm{~cm} / \mathrm{s}$ ) in the group with subsequent ISR (5.9 vs. $33.3 \%, p<0.001$ ) compared to those without ISR (see Table 1).

\section{Independent predictors for ISR}

To identify independent predictors for an ISR, a multiple binominal regression analysis was performed including all variables, which were imbalanced $(p<0.1$ see Table 1$)$ within the univariate analysis (hyperlipidemia, peripheral occlusive artery disease, CEA restenosis, contralateral ICA occlusion/stenosis $\geq 70 \%$, residual stenosis after CAS as detected by PSV $\geq 120 \mathrm{~cm} / \mathrm{s} 1-3$ days after CAS). The strongest statistically significant predictor for the development of a subsequent ISR after multiple regression analysis was a contralateral carotid occlusion (OR 10.11, 95\% CI 2.06-49.63, $p=0.004$ ), a CEA restenosis (OR $8.87,95 \%$ CI $1.68-46.84, p=0.010$ ) as an indication for the elective CAS and a postprocedural carotid duplex ultrasound with an elevated PSV $\geq 120 \mathrm{~cm} / \mathrm{s}$ indicating a residual low-grade stenosis (OR 6.33, 95\% CI 1.27-31.44, $p=0.024)$.

\section{Discussion}

Within the current prospective single-center long-term CAS surveillance we observed significantly more clinical complications (stroke or death) in patients who developed an ISR during follow-up compared to those without ISR. Moreover, a contralateral carotid occlusion, a CAS intervention of a restenosis after CEA and a postprocedural PSV $>120 \mathrm{~cm} / \mathrm{s}$ on duplex sonography indicating a residual low-grade stenosis after CAS could be identified to be independent risk factors for the development of an ISR during follow-up. Against the background of the clinical impact of an ISR, we recommend a tight clinical and ultrasonographic long-term follow-up of patients treated with CAS, especially in those with the aforementioned clinical characteristics.

In the past few years, CAS has frequently been used as an alternative to CEA for the treatment of a carotid artery stenosis yet randomized controlled trials have recently failed to prove a clear benefit in favor for a CAS intervention [1-4]. Especially the long-term benefit of a CAS procedure is currently debated because the CAS data presented to date have reported concerning results [8, 9]. However, according to the current literature a CAS intervention is thought to be effective in younger patients, because two meta-analyses comparing the complications of CAS and CEA showed a trend towards a favorable outcome in patients aged $<70$ years for those patients treated with CAS [1, 4]. On the other hand, within the recently published long-term results of the SPACE and EVA-3S trials, the incidence of an ISR after 2 years diagnosed with duplex sonography was significantly higher after a CAS intervention compared to CEA (10.7 vs. $4.6 \%, p=0.009$ and 12.5 vs. $5.0 \% ; p=0.02)[8,10]$. In those trials the higher incidence of ISR during follow-up was not found to have an impact on clinical complication rates. Therefore, it has been postulated that restenosis might be a relatively benign pathology [9]. In contrast, our results support the notion that the occurrence of ISR among other factors could endanger the long-term efficacy and safety of CAS, because the long-term risks for stroke or death were significantly higher in patients with an ISR. Against the background that a CAS intervention might be beneficial especially in younger patients, these results should be taken into account for the patient's individual treatment advice.

One reason for the higher incidence of clinical complications during medium-term follow-up within our singlecenter experience might be the less well-controlled cardiovascular risk factors after hospital discharge, with, e.g., the lack of routinely scheduled blood samples to adjust current medication such as statins or antidiabetic drugs in our outpatient clinic. The current setting may however reflect common everyday practice in real life and might therefore not necessarily be comparable to the well-structured settings of randomized controlled trials [3, 4, 8]. Moreover, the positive patient selection favoring the compliant and well-educated ones in randomized 
controlled trials has to be taken into account and could explain the disparity in stroke and ISR rates.

As could be expected, the number of re-interventions (8/ $12 ; 66.7 \%$ ) was higher within the patients with high-grade restenosis in our study. Although no clinical periprocedural complications occurred during routinely scheduled reinterventions within the aforementioned patients, the variety of different treatment strategies selected to engage the ISR ( 3 re-CAS, 4 PTA and 1 bypass surgery) reflects the fact that an overall accepted treatment strategy for ISR has not yet been established. As within our series, surgical treatment of ISR remained an exception in the reviewed literature because it is technically demanding and can be associated with periprocedural complications [11]. Currently, in most of the cases a PTA or re-CAS is performed [12]. This further highlights the clinical importance of identifying independent risk factors to be able to detect those patients during clinical routine and leading to a thorough long-term sonographic follow-up.

Current data suggest that ISR frequently occurs during the first year of follow-up [5], which is corroborated within the current patient cohort: ISR was observed after a median follow-up of 8.6 months (IQR 3.4-17.3). Not surprisingly, an insufficient result after CAS with elevated PSV $>120 \mathrm{~cm} / \mathrm{s}$ within the stent as detected with duplex ultrasound was found to be associated with subsequent ISR. This is in line with previous studies and may be due to heavily calcified plaques, which made it difficult to establish an appropriate stent positioning without residual narrowing [13, 14]. Interestingly, in a study with 563 patients, Randall et al. found that a residual stenosis of $>50 \%$ after CAS is associated with an increased risk of ipsilateral stroke in the long run [15]. Therefore, pursuing an optimal stent deployment during CAS seems to be a worthwhile aim, although it is known that an aggressive postdilation bears the risk of distal embolization. It could also result in microvascular injury, which may contribute to an aggravation of inflammatory processes that finally promote neointimal hyperplasia and ISR [7, 16].

Currently, CAS is a recommended treatment alternative for patients with CEA restenosis, because a subsequent CEA bears a higher periprocedural risk than the initial operation [17, 18]. However, in line with our current results, which have identified a previous CEA as an independent risk factor for the development of ISR after CAS, previous authors noted that there may be a higher risk of developing an ISR after stenting of postendarterectomy arteries [18-20]. These findings emphasize that patients with restenosis after CEA and a second CAS intervention are prone to develop a second restenosis.

Another risk factor for ISR identified in this study is the presence of a contralateral carotid artery occlusion. Although higher blood flow velocities in a carotid artery are a well-known phenomenon in case of a contralateral carotid occlusion [21], the restenosis could be confirmed in four of five patients within our patient cohort during conventional angiography (in one patient a confirmation was not possible because of imaging artifacts during contrast-enhanced CT angiography). These imaging results argue against the possibility that an ISR in these patients might be based solely on a false-positive ultrasound measurement triggered by artificially elevated flow accelerations caused by the contralateral carotid occlusion. According to the current literature, some authors state that CAS would be the favorable treatment option in patients with a contralateral carotid artery occlusion [17, 22], because periprocedural complications may be higher during CEA [23]. Nevertheless, the possibility of an elevated flow, which might overestimate a possible ISR in patients with contralateral occlusion, should be kept in mind by clinicians, and even more so the higher incidence of ISR when offering CAS as a treatment option to this patient subgroup.

Despite the strengths of the current study we are well aware of certain limitations: Because of the retrospective analysis of the prospectively recorded data an ascertainment bias cannot be ruled out, although $>90 \%$ of all patients had been followed up. The lack of generally valid ultrasound criteria for the detection of ISR [5, 6] may have led to different rates of ISR, although we adopted our criteria in accordance to the current literature.

\section{Conclusions}

ISR after CAS occurs frequently within the first year of follow-up and is associated with a higher risk of clinical complications. Considering that a CAS intervention is frequently used as an alternative to CEA especially in patients $<70$ years, a strict and long-term follow-up is warranted. Especially patients with the presence of a contralateral carotid artery occlusion treated because of a CEA restenosis or with an insufficient postprocedural result (PSV $>120 \mathrm{~cm} / \mathrm{s}$ ) are prone to develop an ISR. With respect to the clinical relevance of an ISR and the lack of a commonly accepted treatment strategy, all efforts should be made to carefully follow-up especially these patient subgroups. Duplex sonography with adopted local criteria to identify ISR should be used as a non-invasive, inexpensive follow-up modality after CAS.

\section{Conflicts of interest None.}

Open Access This article is distributed under the terms of the Creative Commons Attribution License which permits any use, distribution, and reproduction in any medium, provided the original author(s) and the source are credited. 


\section{References}

1. Economopoulos KP, Sergentanis TN, Tsivgoulis G, Mariolis AD, Stefanadis C (2011) Carotid artery stenting versus carotid endarterectomy: a comprehensive meta-analysis of short-term and long-term outcomes. Stroke 42:687-692

2. Ederle J, Dobson J, Featherstone RL, Bonati LH, van der Worp HB, de Borst GJ, Lo TH, Gaines P, Dorman PJ, Macdonald S, Lyrer PA, Hendriks JM, McCollum C, Nederkoorn PJ, Brown MM (2010) Carotid artery stenting compared with endarterectomy in patients with symptomatic carotid stenosis (International Carotid Stenting Study): an interim analysis of a randomised controlled trial. Lancet 375:985-997

3. Brott TG, Hobson RW, Howard G, Roubin GS, Clark WM, Brooks W, Mackey A, Hill MD, Leimgruber PP, Sheffet AJ, Howard VJ, Moore WS, Voeks JH, Hopkins LN, Cutlip DE, Cohen DJ, Popma JJ, Ferguson RD, Cohen SN, Blackshear JL, Silver FL, Mohr JP, Lal BK, Meschia JF (2010) Stenting versus endarterectomy for treatment of carotid-artery stenosis. N Engl J Med 363:11-23

4. Bonati LH, Dobson J, Algra A, Branchereau A, Chatellier G, Fraedrich G, Mali WP, Zeumer H, Brown MM, Mas JL, Ringleb PA (2010) Short-term outcome after stenting versus endarterectomy for symptomatic carotid stenosis: a preplanned meta-analysis of individual patient data. Lancet 376:1062-1073

5. Gröschel K, Riecker A, Schulz JB, Ernemann U, Kastrup A (2005) Systematic review of early recurrent stenosis after carotid angioplasty and stenting. Stroke 36:367-373

6. Nederkoorn PJ, Brown MM (2009) Optimal cut-off criteria for duplex ultrasound for the diagnosis of restenosis in stented carotid arteries: review and protocol for a diagnostic study. BMC Neurol 9:36

7. Wasser K, Schnaudigel S, Wohlfahrt J, Psychogios MN, Knauth M, Gröschel K (2011) Inflammation and in-stent restenosis: the role of serum markers and stent characteristics in carotid artery stenting. PLoS One 6:e22683

8. Arquizan C, Trinquart L, Touboul PJ, Long A, Feasson S, Terriat B, Gobin-Metteil MP, Guidolin B, Cohen S, Mas JL (2011) Restenosis is more frequent after carotid stenting than after endarterectomy: the EVA-3S study. Stroke 42:1015-1020

9. Naylor AR (2008) Stenting versus endarterectomy: the debate continues. Lancet Neurol 7:862-864

10. Eckstein HH, Ringleb P, Allenberg JR, Berger J, Fraedrich G, Hacke W, Hennerici M, Stingele R, Fiehler J, Zeumer H, Jansen O (2008) Results of the Stent-Protected Angioplasty versus Carotid Endarterectomy (SPACE) study to treat symptomatic stenoses at 2 years: a multinational, prospective, randomised trial. Lancet Neurol 7:893-902

11. Reichmann BL, van Laanen JH, De Vries JP, Hendriks JM, Verhagen HJ, Moll FL, de Borst GJ (2011) Carotid endarterectomy for treatment of in-stent restenosis after carotid angioplasty and stenting. J Vasc Surg 54:87-92

12. van Haaften AC, Bots ML, Moll FL, de Borst GJ (2010) Therapeutic options for carotid in-stent restenosis: review of the literature. J Vasc Interv Radiol 21:1471-1477

13. Khan MA, Liu MW, Chio FL, Roubin GS, Iyer SS, Vitek JJ (2003) Predictors of restenosis after successful carotid artery stenting. Am J Cardiol 92:895-897

14. Cosottini M, Michelassi MC, Bencivelli W, Lazzarotti G, Picchietti S, Orlandi G, Parenti G, Puglioli M (2010) In stent restenosis predictors after carotid artery stenting. Stroke Res Treat. pii:864724.:864724

15. Randall MS, McKevitt FM, Kumar S, Cleveland TJ, Endean K, Venables GS, Gaines PA (2010) Long-term results of carotid artery stents to manage symptomatic carotid artery stenosis and factors that affect outcome. Circ Cardiovasc Interv 3:50-56

16. Clark DJ, Lessio S, O'Donoghue M, Tsalamandris C, Schainfeld R, Rosenfield K (2006) Mechanisms and predictors of carotid artery stent restenosis: a serial intravascular ultrasound study. J Am Coll Cardiol 47:2390-2396

17. Kastrup A, Gröschel K (2007) Carotid endarterectomy versus carotid stenting: an updated review of randomized trials and subgroup analyses. Acta Chir Belg 107:119-128

18. AbuRahma AF, Abu-Halimah S, Bensenhaver J, Nanjundappa A, Stone PA, Dean LS, Keiffer T, Emmett M, Tarakji M, AbuRahma Z (2009) Primary carotid artery stenting versus carotid artery stenting for postcarotid endarterectomy stenosis. J Vasc Surg 50:1031-1039

19. Setacci C, Pula G, Baldi I, de Donato G, Setacci F, Cappelli A, Pieraccini M, Cremonesi A, Castriota F, Neri E (2003) Determinants of in-stent restenosis after carotid angioplasty: a casecontrol study. J Endovasc Ther 10:1031-1038

20. Zhou W, Lin PH, Bush RL, Peden EK, Guerrero MA, Kougias P, Lumsden AB (2006) Management of in-sent restenosis after carotid artery stenting in high-risk patients. J Vasc Surg 43:305-312

21. Busuttil SJ, Franklin DP, Youkey JR, Elmore JR (1996) Carotid duplex overestimation of stenosis due to severe contralateral disease. Am J Surg 172:144-147 (discussion 147-148)

22. Hobson RW, Mackey WC, Ascher E, Murad MH, Calligaro KD, Comerota AJ, Montori VM, Eskandari MK, Massop DW, Bush RL, Lal BK, Perler BA (2008) Management of atherosclerotic carotid artery disease: clinical practice guidelines of the Society for Vascular Surgery. J Vasc Surg 48:480-486

23. Gasecki AP, Eliasziw M, Ferguson GG, Hachinski V, Barnett HJ (1995) Long-term prognosis and effect of endarterectomy in patients with symptomatic severe carotid stenosis and contralateral carotid stenosis or occlusion: results from NASCET. North American Symptomatic Carotid Endarterectomy Trial (NASCET) Group. J Neurosurg 83:778-782 